\title{
Technical note: Identification of Prototheca species from bovine milk samples by PCR-single strand conformation polymorphism
}

\author{
P. Cremonesi, ${ }^{\star 1}$ F. Pozzi, $†$ M. Ricchi, $\neq$ B. Castiglioni, ${ }^{*}$ M. Luini, $†$ and S. Chessa* \\ *Istituto di Biologia e Biotecnologia Agraria, Consiglio Nazionale delle Ricerche (CNR), via Einstein, 26900 Lodi, Italy \\ †Istituto Zooprofilattico Sperimentale della Lombardia e dell'Emilia - Sezione di Lodi, via Einstein, 26900 Lodi, Italy \\ łlstituto Zooprofilattico Sperimentale della Lombardia e dell'Emilia - Sezione di Piacenza, Strada Vicinale Faggiola, \\ 29027 Podenzano, Piacenza, Italy
}

\begin{abstract}
We report the development of a PCR-single strand conformation polymorphism (SSCP) method to identify Prototheca spp. responsible for bovine mastitis: P. zopfii and $P$. blaschkeae. The method was set up using reference strains belonging to P. zopfii genotype 1, P. zopfii genotype 2, and P. blaschkeae as target species and $P$. stagnora, and $P$. ulmea as negative controls. The assay was applied on 50 isolates of Prototheca spp. isolated from bovine mastitic milk or bulk-tank milk samples, and all isolates were identified as P. zopfii genotype 2 . We conclude that the described PCR-SSCP approach is accurate, inexpensive, and highly suitable for the identification of $P$. zopfii genotype 2 on field isolates but also directly on milk, if preceded by a specific DNA extraction method.
\end{abstract}

Key words: Prototheca spp., Prototheca zopfii, PCRsingle strand conformation polymorphism, mastitis

\section{Technical Note}

Algae of the genus Prototheca are aerobic, achlorophyllic, nonphotosynthetic, round-to-ovoid, yeast-like, unicellular algae. They are ubiquitous organisms that live saprophytically on decaying organic matter in fresh and marine water, fish tanks, swimming pools, ponds, soil, vegetables, and meat products (Pore et al., 1983). At present, 6 species are assigned to the genus Prototheca: P. zopfii, P. wickerhamii, P. stagnora, P. ulmea, P. blaschkeae, and $P$. cutis sp. nov., the last being a novel species responsible for human dermatitis (Satoh et al., 2010). A seventh species, P. moriformis, is not currently accepted because of its biochemical and genetic resemblance to $P$. zopfii and its high intraspecific heterogeneity (Roesler et al., 2006). Based on its biochemical, serological, and 18S ribosomal (r)

Received May 30, 2012.

Accepted July 29, 2012.

${ }^{1}$ Corresponding author: cremonesi@ibba.cnr.it
DNA sequencing data, $P$. zopfii is differentiated into 2 genotypes: 1 and 2 (Roesler et al., 2006). The species $P$. stagnora and $P$. ulmea have not so far been associated with any disease. Recently, $P$. zopfii genotype 1 was related to cases of bovine mastitis (Ahrholdt et al., 2012), and P. wickerhamii, P. zopfii genotype 2, P. blaschkeae, and $P$. cutis have been associated with both human and animal diseases (Roesler et al., 2006; Ricchi et al., 2010; Satoh et al., 2010). Prototheca wickerhamii is the most common species recovered in human diseases, but it was recently isolated in goats with multifocal ulcerative lesions of the mucosa of nostrils and surrounding skin (Macedo et al., 2008; Camboim et al., 2011). On the other hand, P. zopfii was found to be more pathogenic and virulent in immunosuppressed mice, laboratory animals, dog, cats, and cattle (Möller et al., 2007; Osumi et al., 2008).

Prototheca was first linked to mastitis in dairy cows in 1952, but its first description as an agent of bovine mastitis dates from 1985 (Blaschke-Hellmessen et al., 1985). Today, the number of cases of bovine mastitis caused by this genus is increasing worldwide. Almost all Prototheca isolates from bovine mastitis in Italy, Germany, Portugal, Poland, Japan, and China came from $P$. zopfii genotype 2, suggesting that it is the principal causative agent, whereas $P$. blaschkeae was found to cause bovine mastitis only sporadically (Möller et al., 2007; Marques et al., 2008; Osumi et al., 2008; Kishimoto et al., 2010; Ricchi et al., 2010; Jagielski et al., 2011; Gao et al., 2012).

Protothecal bovine infection is slowly progressive and often subclinical, making it difficult to recognize early. It leads to limited to mild changes in milk, with an increase in SCC, reduced milk production, and a thin watery milk secretion containing white flakes. Treatment for protothecal infections remains controversial; various treatment regimens have been attempted, but without consistency in the clinical responses (Buzzini et al., 2008). No easy way exists to prevent Prototheca infections; on farms where it is present, Prototheca is widely spread. The detection of individual cases of pro- 
tothecal mastitis in some herds can indicate a serious problem that affects a significant percentage of cows.

Different molecular techniques have been proposed to identify Prototheca species: genotype-specific PCR (Roesler et al., 2006; Osumi et al., 2008) and RFLP analysis (Möller et al., 2007) distinguish P. blaschkeae and $P$. zopfii but are unable to differentiate among the other Prototheca species. The 2-step real-time quantitative PCR reaction followed by DNA resolution melting analysis recently developed (Ricchi et al., 2011) can distinguish among different species and genotypes. However, the protocol described requires $48 \mathrm{~h}$ of cell growth and 2 steps of quantitative PCR and resolution melting analysis, the first distinguishing among $P$. zopfii genotype 1, P. zopfii genotype 2, and P. blaschkeae, and the second to identify $P$. ulmea, $P$. stagnora, and $P$. wickerhamii. Moreover, the $18 \mathrm{~S}$ rDNA sequencing described (Marques et al., 2008) requires $48 \mathrm{~h}$ of cell growth, PCR amplifications, sequencing, and bioinformatic analyses to identify the species and is therefore extremely time consuming.

The aim of this study was to develop a rapid method to differentiate $P$. zopfii genotype 2, responsible for bovine mastitis, from other microalgae. Polymerase chain reaction-SSCP is a rapid, easy, and highly reproducible technique for detection and genotyping of SNP, small insertion-deletions, and DNA rearrangements. It permits identification of nucleotide substitutions because different intra-molecular pairings of the bases result in a conformation polymorphism of the single-stranded DNA after denaturation. The different conformations correspond to different migrations during electrophoresis in a vertical polyacrylamide gel. The technique has been used successfully to analyze polymorphisms in human, animal, and microbial DNA (Kakavas et al., 2008; Küpper et al., 2010; Xu et al., 2010) and was therefore chosen to analyze Prototheca DNA. This is the first time that PCR-SSCP has been applied to distinguish these unicellular algae.

To optimize the PCR-SSCP protocol, a few reference strains belonging to both pathogenic ( $P$. zopfii genotype 2, Istituto Zooprofilattico Sperimentale della Lombardia e dell'Emilia (IZSLER) 219509; P. blaschkeae, IZSLER 161681) and nonpathogenic species (P. zopfii genotype 1, IZSLER 308527; P. stagnora, ATCC 16528; P. ulmea, ATCC 50112) were used. Prototheca zopfii genotype 2, IZSLER 219509 and P. zopfii genotype 1, IZSLER 308527 were described in Ricchi et al. (2011), whereas P. blaschkeae, IZSLER 161681 was isolated from bulk tank milk sample and the strain were identified by $18 \mathrm{~S}$ rDNA sequencing.

At the same time, 40 bovine mastitic milk and 10 bulk-tank milk samples from Italian herds located in the Lombardy region were used to validate the protocol developed. The 50 milk samples were used both for direct Prototheca DNA extraction and to obtain isolates for microbiological analyses. Both isolates and reference strains were aerobically cultured on Sabouraud dextrose agar (Biogenetics, Padova, Italy) and brain heart infusion agar (bioMérieux, Bagno a Ripoli, Italy) plates at $37^{\circ} \mathrm{C}$ for $48 \mathrm{~h}$.

If not otherwise specified, all chemicals for DNA extraction and the PCR-SSCP protocol were from Sigma Aldrich (Milan, Italy). Genomic DNA of the microalga was extracted from the Prototheca isolates and the milk samples, using the procedure described in Cremonesi et al. (2006) with minor modifications. Briefly, for the isolates and reference strains, a few colonies collected by scraping a plate were diluted in $400 \mu \mathrm{L}$ of lysis buffer $(3 \mathrm{~mol} / \mathrm{L}$ guanidine thiocyanate, $20 \mathrm{mmol} / \mathrm{L}$ EDTA, $10 \mathrm{mmol} / \mathrm{L}$ Tris-HCl, $\mathrm{pH}$ 6.8, $40 \mathrm{mg} / \mathrm{mL}$ Triton X-100, $10 \mathrm{mg} / \mathrm{mL}$ dithiothreitol) and vortexed for $30 \mathrm{~s}$ to obtain an emulsified solution. After addition of $500 \mu \mathrm{L}$ of binding solution ( $40 \mathrm{mg} / \mathrm{mL}$ silica from Sigma Aldrich, directly suspended in the lysis buffer), the sample was incubated for $5 \mathrm{~min}$ at 20 to $24^{\circ} \mathrm{C}$. After centrifugation for $30 \mathrm{~s}$ at $550 \times \mathrm{g}$, the supernatant was discarded and the silica-DNA pellet obtained was subsequently washed twice with $200 \mu \mathrm{L}$ of lysis buffer, twice with $200 \mu \mathrm{L}$ of washing solution (25\% absolute ethanol, $25 \%$ isopropanol, $100 \mathrm{mmol} / \mathrm{L} \mathrm{NaCl}, 10 \mathrm{mmol} / \mathrm{L}$ Tris- $\mathrm{HCl}$, $\mathrm{pH} 8$ ) and once with $200 \mu \mathrm{L}$ of absolute ethanol. After every washing and vortexing, the silica-DNA pellet was centrifuged for $30 \mathrm{~s}$ at $550 \times g$ and the supernatant was discarded. The pellet was then vacuum-dried at $56^{\circ} \mathrm{C}$ in a heat block for $10 \mathrm{~min}$. After addition of $100 \mu \mathrm{L}$ of elution buffer $(10 \mathrm{mmol} / \mathrm{L}$ Tris-HCl, $\mathrm{pH} 8,1 \mathrm{mmol} / \mathrm{L}$ EDTA), the silica-DNA pellet was gently vortexed and incubated for $15 \mathrm{~min}$ at $65^{\circ} \mathrm{C}$. After a 5 -min centrifugation at $550 \times g$, the supernatant containing the DNA was recovered and stored at $-20^{\circ} \mathrm{C}$.

For milk samples, the DNA was extracted following the procedure described by Cremonesi et al. (2006); the only change made to the protocol described was to increase, from step 2 to 6 , the centrifugation speed up to $550 \times g$. The DNA was spectrophotometrically quali-quantified using the NanoDrop ND-1000 UVVis Spectrophotometer (NanoDrop Technologies Inc., Wilmington, DE). The concentration (mean $\pm \mathrm{SD}$ ) of DNA extracted from the strains and mastitic bulk-tank milk samples was $60 \pm 15 \mathrm{ng} / \mu \mathrm{L}$ with an absorbance ratio at $260: 280 \mathrm{~nm}\left(\Delta_{260 / 280}\right)$ of $1.77 \pm 0.1$, showing the efficiency of this procedure to reduce, during sample preparation, the presence of inhibitory substances precluding DNA amplification.

Two regions of the $18 \mathrm{~S}$ rDNA containing the mutations distinguishing the above-mentioned species were targeted for primer design using Primer3 software (http:// 
frodo.wi.mit.edu/primer3/; Rozen and Skaletsky, 2000). Based on published sequences (GenBank accession numbers AY973040 for P. zopfii genotype 1, AY940456 for P. zopfii genotype 2, AB096930 for P. stagnora, X74003 for P. wickerhamii, AB096929 for P. ulmea, AY973041 for P. blaschkeae, and AB470468 for P. cutis), 2 pairs of primers amplifying 2 fragments of $313 \mathrm{bp}$ [forward $(\mathrm{Fw})$ $5^{\prime}$ TGCTGCAGTTAAAAAGCTCGT $3^{\prime}$ and reverse $(\mathrm{Rv}) 5^{\prime}$ TCCAAGAATTTCACCTCTGACA $\left.3^{\prime}\right]$ and $290 \mathrm{bp}\left(\mathrm{Fw} 5^{\prime}\right.$ AGCCTGCGGCTTAATTTGAC $3^{\prime}$ and Rv 5' CGGCCCAGAACATCTAAGG 3'), respectively, were designed (Figure 1). All PCR reactions were carried out in a GeneAmpPCR System 2700 (Applied Biosystems, Foster City, CA) in 0.2-mL tubes containing $12.5 \mu \mathrm{L}$ of $2 \times$ PCR Master Mix (Fermentas, M-Medical
SRL, Milan, Italy), 20 pmol of each primer, $30 \mathrm{ng}$ of extracted DNA, and sterile water in a total reaction volume of $25 \mu \mathrm{L}$. A pre-PCR step was run at $94^{\circ} \mathrm{C}$ for 5 min followed by 30 PCR cycles under the following conditions: denaturation at $94^{\circ} \mathrm{C}$ for $1 \mathrm{~min}$, annealing at $56^{\circ} \mathrm{C}$ for $1 \mathrm{~min}$, and extension at $72^{\circ} \mathrm{C}$ for $1 \mathrm{~min}$. After the final cycle, the preparation was kept at $72^{\circ} \mathrm{C}$ for $10 \mathrm{~min}$ to complete the reaction.

The 2 PCR products were analyzed by means of SSCP, loading the products in the gel both separately and together in the same slot. In both cases, $6 \mu \mathrm{L}$ of PCR product was added to $8 \mu \mathrm{L}$ of denaturation solution $(0.05 \%$ of xylenecyanol, $0.05 \%$ of bromophenol blue, 0.02 $M$ EDTA in deionized formamide), subjected to heat denaturation at $95^{\circ} \mathrm{C}$ for $8 \mathrm{~min}$, and then im-

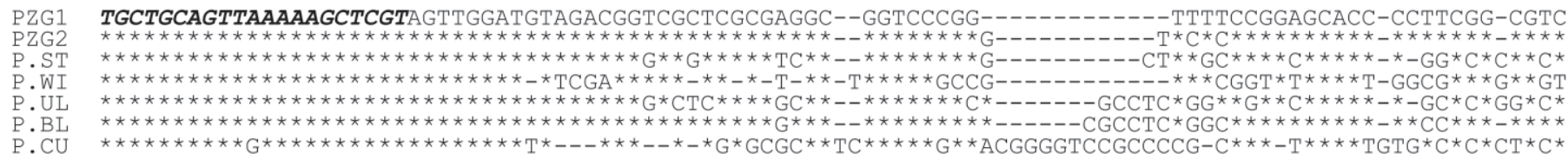

PZG1 CTCTG--GCTGGCGAGCGTCGCGG-CT-GGGCTTCAC-CGCTCGGCCGCGGGCCTCGCCGTGGTTACTTTGAGTAAATTGGAGTGTTCAAAGCAGGCGTT

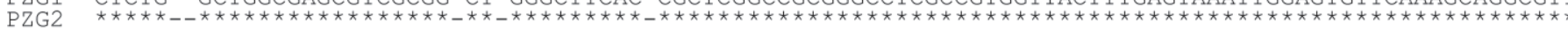

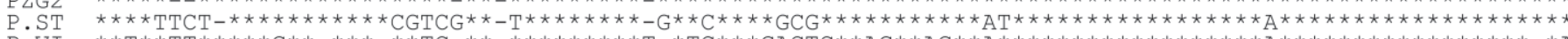

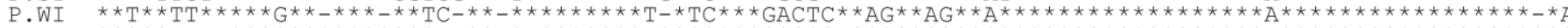

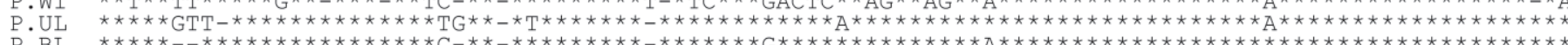

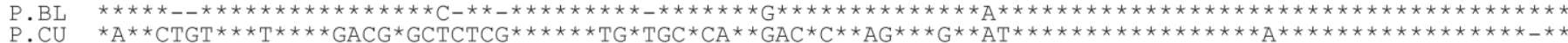

PZG1 -CGCC-GTGGACACGTTAGCATGGAATAACGGCACAGGACTCTGGCCTGTCCCGTTGGTCTGCAGGACCGGAGTAATGATGAAGAGGAACAGTCGGGGGC

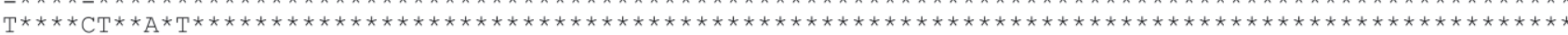

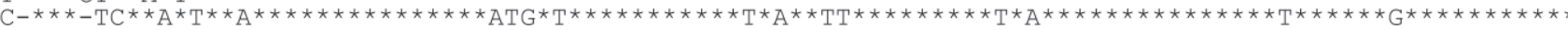

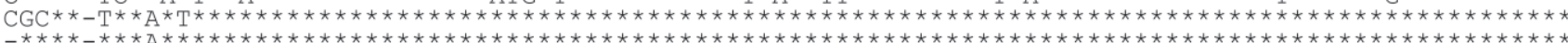

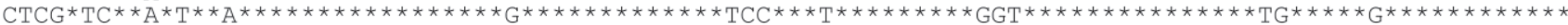

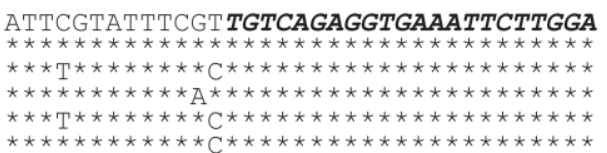

AGCCTGCGGCTTAATTTGACTCAACACGGGGAAACTTACCAGGTCCAGACATGGCGAGGATTGACAGATTGATAGCTCTTTCTTGATTCCATGGGTGGTG 等

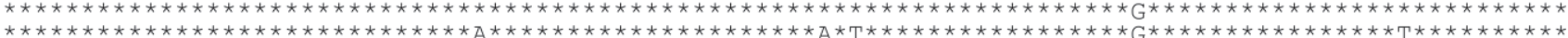

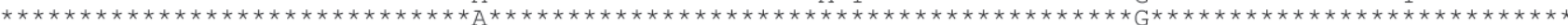

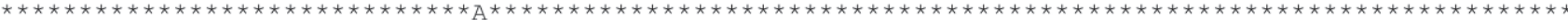

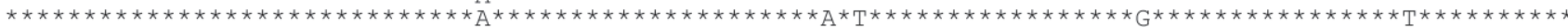

GTGCATGGCCGTTCTTAGTTGGTGGGTTGCCTTGTCAGGTTGATTCCGGTAACGAACGAGACCTCGGCCTGCTAACTAGCTTCGGGGGGCCTTGGCCCCG

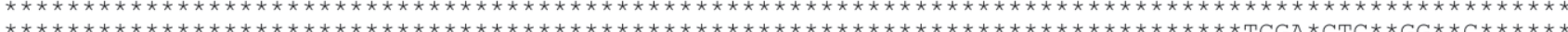

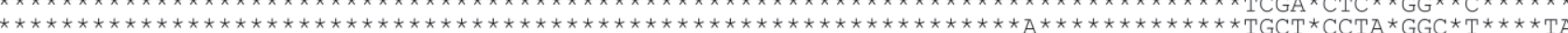

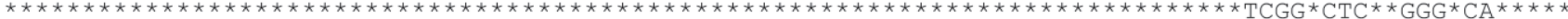

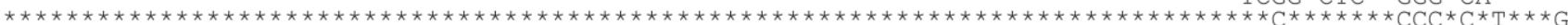

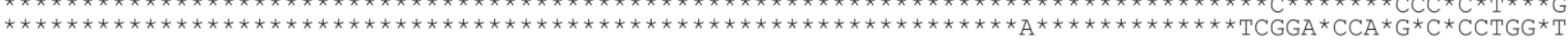

CCGACG-----CTTCTTAGAGGGACTGCTGGCGTT-CCAGCCAGCGGAAGCGCGAGGCGATAACAGGTCTGTGATGCCCTTAGATGTTCTGGGCCG

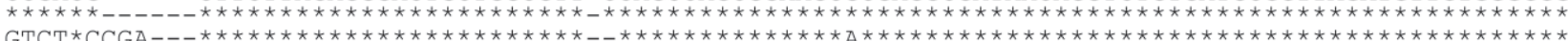

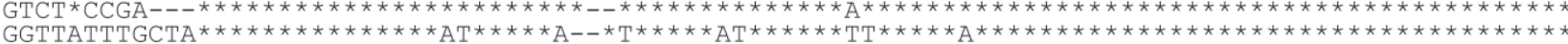
GGTC*CTCCGAGGTC*CTCCGA-

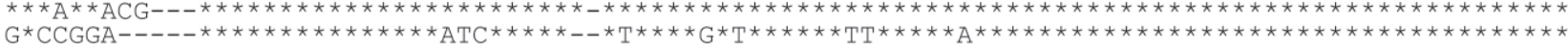

Figure 1. Sequence alignment of the 6 species assigned to the genus Prototheca. The first alignment corresponds to the 18S ribosomal (r) DNA 313-bp amplified fragment and the second to the $18 \mathrm{~S}$ rDNA 290-bp amplified fragment. Primer sequences are in bold italics. PZG1 $=P$. zopfii genotype 1 (GenBank accession no. AY973040); PZG2 = P. zopfii genotype 2 (AY940456); P.ST = P. stagnora (AB096930); P.WI = P. wickerhamii (X74003); P.UL = P. ulmea (AB096929); P.BL = P. blaschkeae (AY973041); P.CU = P. cutis (AB470468). 
mediately chilled on ice. To find the best conditions for separating the different Prototheca species and guaranteeing the highest reproducibility of the method, different conditions of acrylamide concentration (10 and $12 \%$ ), crosslinking (2.5 and $3.3 \%$ ), and glycerol concentration $(1.5,0.5$, and $0 \%)$ were tested. Three different run temperatures $\left(5,9\right.$, and $\left.12^{\circ} \mathrm{C}\right)$ were tested. The final protocol consisted of a $10 \%$ polyacrylamide gel with an acrylamide:bisacrylamide ratio of 37.5:1, without glycerol, in $0.5 \times$ Tris-borate-EDTA buffer in a electrophoretic run performed at $220 \mathrm{~V}$ and $9^{\circ} \mathrm{C}$ for 15 $\mathrm{h}$ in a Penguin TM Dual Gel Water-Cooled Electrophoresis System (Owl Scientific Inc., Woburn, MA). Bands were visualized by silver staining.

The PCR products of 6 of the 50 isolates of Prototheca were sequenced by Primm srl (Milan, Italy) using the primers described above to confirm the results. The nucleotide sequences were analyzed with Bioedit software (Hall, 1999).

The simultaneous analysis of 2 different regions of $18 \mathrm{~S}$ rDNA allowed discrimination of all Prototheca species analyzed. In particular, for the 313-bp fragment, the difference in migration between $P$. zopfii genotype 2 and $P$. blaschkeae was almost undetectable, as was that between P. stagnora and P. ulmea, whereas P. zopfii genotype 1 was easily discriminated from the other 4 species (Figure 2). The 290-bp fragment produced 5 well-differentiated patterns, each one corresponding to the different species analyzed, the order of migration from slowest to fastest being P. stagnora, P. blaschkeae, P. ulmea, $P$. zopfii genotype 1 , and $P$. zopfii genotype 2. Loading both the 313- and the 290-bp fragments, the 5 species analyzed yielded 5 specific and easy-todistinguish patterns. A small change in migration order compared with the 290-bp fragment only migration was seen because the overall migration pattern of $P$. ulmea was slower than that of P. blaschkeae.

The assay was evaluated by analyzing 50 Prototheca species strains isolated from individual bovine mastitic or bulk-tank milk samples from routine diagnostic activity. After isolation on Sabouraud dextrose agar, the strains were identified basing on cultural and microscopic features. Macroscopic large, whitish colonies of the Prototheca colonies were subjected to microscopic examination, showing daisy-like organisms, whereas Gram staining showed large, gram-positive algal bodies with irregular shape.

Moreover, all field strains isolated from Lombardy herds gave the same migration pattern with PCRSSCP and were identified as $P$. zopfii genotype 2. All

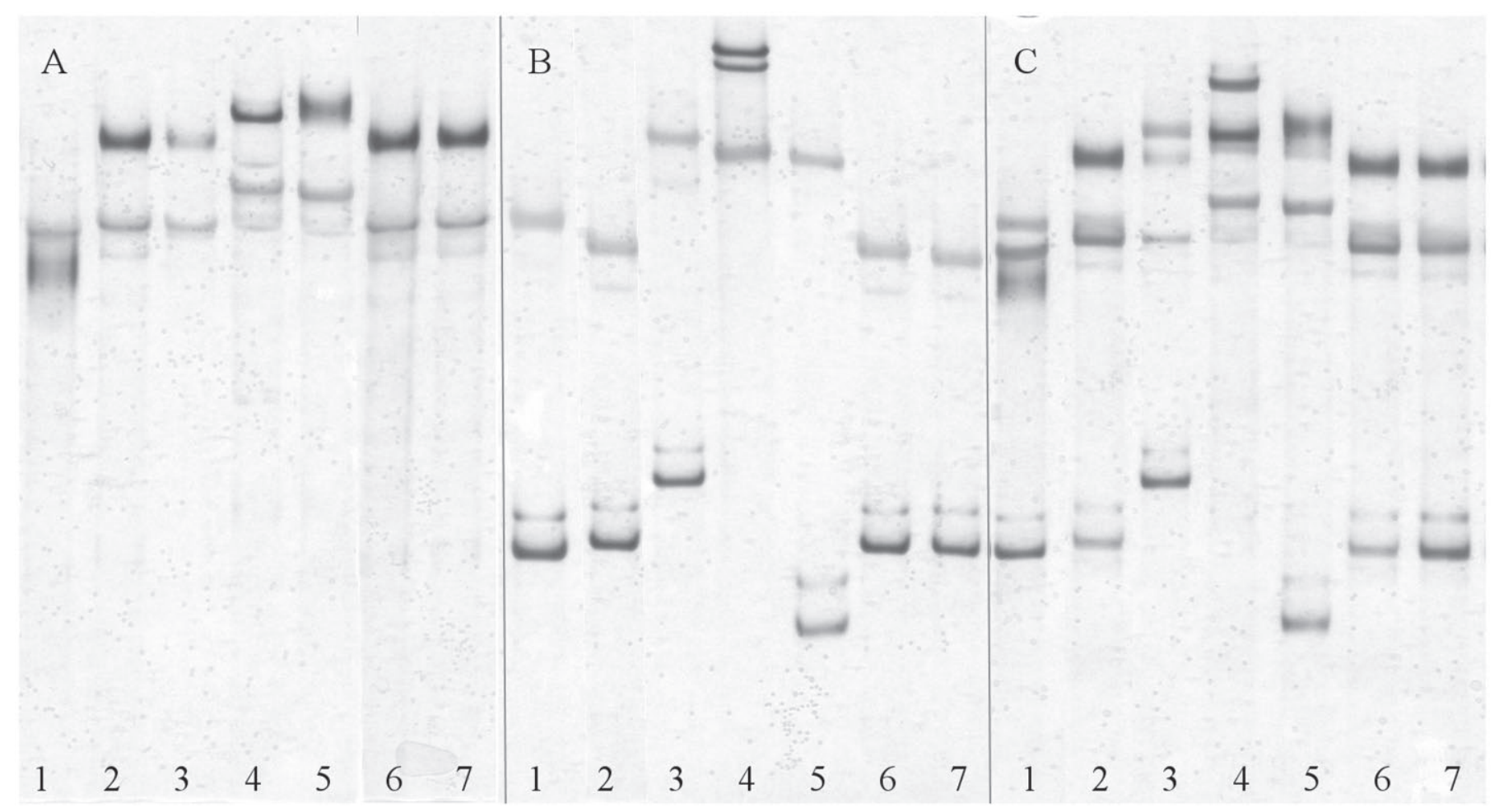

Figure 2. Polymerase chain reaction-single strand conformation polymorphism analysis of the two 18S ribosomal DNA fragments amplified. (A) Migration patterns obtained for the 313-bp fragment; (B) migration patterns obtained for the 290-bp fragment; (C) migration patterns of both fragments loaded in the same slot. Lane $1=$ Prototheca zopfii genotype 1 ; lane $2=$ P. zopfii genotype 2 ; lane $3=$ Prototheca blaschkeae; lane $4=$ Prototheca stagnora; lane $5=$ Prototheca ulmea; lanes 6 and $7=$ field samples corresponding to P. zopfii genotype 2 . 
samples were tested at least 3 times, always with the same result. Microbiological and sequencing data confirmed their identification.

The PCR amplification of bacterial DNA extracted directly from milk can be unsuccessful because of the presence of many inhibitors in the biological samples and the difficulty in breaking the cell membrane. As previously described (Cremonesi et al., 2006), increasing the concentration of guanidine thiocyanate and the quantity of lysis buffer solution in the extraction protocol allowed us to obtain DNA optimized for the downstream analysis. In addition, for the Prototheca DNA extraction, the same modifications, together with an increase in centrifugation speed, allowed successful amplification of all samples directly extracted from bulk tank milk, confirming the absence of potential inhibitory factors (i.e., fats, proteinases, high concentration of $\mathrm{Ca}^{2+}$ ) and the applicability of this protocol without need for enrichment. Today, the only Prototheca spp. gene sequences available for all species are those of $18 \mathrm{~S}$ rRNA. Because this gene is highly conserved, a simple PCR amplification, even if specifically designed based on the available sequences, is not sufficient to distinguish between the different species. Therefore, a further analysis of the amplified fragment is necessary to identify the species responsible for mastitis.

Hayashi (1991) estimated the sensitivity of the PCRSSCP as the probability of detecting a point mutation, finding values of $99 \%$ for fragments from 100 to 300 $\mathrm{bp}$, and $89 \%$ for fragments from 300 to $450 \mathrm{bp}$. According to Nataraj et al. (1999), mutations in 100- to 300-bp fragments are easily detected by SSCP analysis when they have $60 \% \mathrm{GC}$ content but not when they have $40 \%$ GC content. For these reasons, 2 primer pairs amplifying 2 fragments of approximately $300 \mathrm{bp}$ were designed around 2 highly variable DNA regions based on the published sequences. Considering only the amplified fragments of $P$. zopfii genotype 1, the GC content was $58.4 \%$ for the 313-bp fragment and $56.6 \%$ for the 290-bp fragment. Thus, good separation of all the species analyzed was expected. Considering these prerequisites, the most informative fragment should have been the 313-bp fragment, especially as the sequence alignment showed less similarity among species than in the 290-bp fragment. However, the shorter the fragment, the greater the probability of distinguishing even a single nucleotide mutation, thus making the 290bp fragment more useful for species identification. The easily distinguishable migration patterns demonstrate that differences at the genomic level in the analyzed fragments were sufficient to discriminate the 5 species, with the potential also to distinguish $P$. wickerhamii and $P$. cutis.
The analysis of 50 Prototheca spp. strains isolated from individual bovine mastitic or bulk-tank milk samples showed that all were $P$. zopfii genotype 2, confirming the predominance of this genotype as a pathogenic agent of bovine protothecal mastitis, as concluded by other authors (Möller et al., 2007; Marques et al., 2008; Osumi et al., 2008; Kishimoto et al., 2010; Ricchi et al., 2010; Gao et al., 2012).

The PCR-SSCP approach is a technique of great advantage to quickly and economically analyze the genetic variability of the Prototheca species, in particular for identification of $P$. zopfii genotype 2 in field isolates. Moreover, using the specific DNA extraction method described here, the developed assay could be used directly on milk samples. Finally, with this technique, it is possible to identify other Prototheca species such as $P$. stagnora and P. ulmea that cannot be discriminated by conventional PCR assay.

\section{ACKNOWLEDGMENTS}

Research was supported by Mastfield Project "Applicazione di sistemi molecolari innovativi per il controllo in campo delle mastiti bovine", funded by Regione Lombardia, DG Agricoltura.

\section{REFERENCES}

Ahrholdt, J., J. Murugaiyan, R. K. Straubinger, T. Jagielski, and U. Roesler. 2012. Epidemiological analysis of worldwide bovine, canine and human clinical Prototheca isolates by PCR genotyping and MALDI-TOF mass spectrometry proteomic phenotyping. Med. Mycol. 50:234-243.

Blaschke-Hellmessen, R., H. Schuster, and V. Bergmann. 1985. Differenzierung von Varianten bei Prototheca zopfii (Krüger 1894). Arch. Exp. Veterinarmed. 39:387-397.

Buzzini, P., B. Turchetti, E. Branda, M. Goretti, M. Amici, P. E. Lagneau, L. Scaccabarozzi, V. Bronzo, and P. Moroni. 2008. Largescale screening of the in vitro susceptibility of Prototheca zopfii towards polyene antibiotics. Med. Mycol. 46:511-514.

Camboim, E. K. A., F. J. Garino, A. F. M. Dantas, S. V. D. Simões, M. A. Melo, E. O. Azevedo, R. A. Mota, and F. Riet-Correa. 2011. Protothecosis by Prototheca wickerhamii in goats. Mycoses 54:e196-e200.

Cremonesi, P., B. Castiglioni, G. Malferrari, I. Biunno, C. Vimercati, P. Moroni, S. Morandi, and M. Luzzana. 2006. Technical note: Improved method for rapid DNA extraction of mastitis pathogens directly from milk. J. Dairy Sci. 89:163-169.

Gao, J., H. Q. Zhang, J. Z. He, Y. H. He, S. M. Li, R. G. Hou, Q. X. Wu, Y. Gao, and B. Han. 2012. Characterization of Prototheca zopfii associated with outbreak of bovine clinical mastitis in herd of Beijing, China. Mycopathologia 173:275-281.

Hall, T. A. 1999. BioEdit: A user-friendly biological sequence alignment editor and analysis program for Windows 95/98/NT. Nucleic Acids Symp. Ser. 41:95-98.

Hayashi, K. 1991. PCR-SSCP: A simple and sensitive method for detection of mutations in the genomic DNA. Genome Res. 1:34-38.

Jagielski, T., H. Lassa, J. Ahrholdt, E. Malinowski, and U. Roesler. 2011. Genotyping of bovine Prototheca mastitis isolates from Poland. Vet. Microbiol. 149:283-287. 
Kakavas, V. K., P. Plageras, T. A. Vlachos, A. Papaioannou, and V. A. Noulas. 2008. PCR-SSCP: A method for the molecular analysis of genetic diseases. Mol. Biotechnol. 38:155-163.

Kishimoto, Y., R. Kano, H. Maruyama, M. Onozaki, K. Makimura, T. Ito, K. Matsubara, A. Hasegawa, and H. Kamata. 2010. 26S rDNA-Based phylogenetic investigation of Japanese cattle-associated Prototheca zopfii isolates. J. Vet. Med. Sci. 72:123-126.

Küpper, J., S. Chessa, D. Rignanese, A. Caroli, and G. Erhardt. 2010. Divergence at the casein haplotypes in dairy and meat goat breeds. J. Dairy Res. 77:56-62.

Macedo, J. T. S. A., F. Riet-Correa, A. F. M. Dantas, and S. V. D Simões. 2008. Cutaneous and nasal protothecosis in a goat. Vet. Pathol. 45:352-354.

Marques, S., E. Silva, C. Kraft, J. Carvalheira, A. Videira, V. A. R. Huss, and G. Thompson. 2008. Bovine mastitis associated with Prototheca blaschkeae. J. Clin. Microbiol. 46:1941-1945.

Möller, A., U. Truyen, and U. Roesler. 2007. Prototheca zopfii genotype 2: The causative agent of bovine protothecal mastitis? Vet. Microbiol. 120:370-374.

Nataraj, A. J., I. Olivos-Glander, N. Kusukawa, and W. E. Highsmith Jr. 1999. Single-strand conformation polymorphism and heteroduplex analysis for gel-based mutation detection. Electrophoresis 20:1177-1185.

Osumi, T., Y. Kishimoto, R. Kano, H. Maruyama, M. Onozaki, K Makimura, T. Ito, K. Matsubara, and A. Hasegawa. 2008. Prototheca zopfii genotypes isolated from cow barns and bovine mastitis in Japan. Vet. Microbiol. 131:419-423.

Pore, R. S., E. A. Barnett, W. C. Barnes Jr., and J. D. Walker. 1983. Prototheca ecology. Mycopathologia 81:49-62.
Ricchi, M., G. Cammi, C. A. Garbarino, P. Buzzini, G. L. Belletti, and N. Arrigoni. 2011. A rapid real-time PCR/DNA resolution melting method to identify Prototheca species. J. Appl. Microbiol. 110:27-34.

Ricchi, M., M. Goretti, E. Branda, G. Cammi, C. A. Garbarino, B. Turchetti, P. Moroni, N. Arrigoni, and P. Buzzini. 2010. Molecular characterization of Prototheca strains isolated from Italian dairy herds. J. Dairy Sci. 93:4625-4631.

Roesler, U., A. Moller, A. Hensel, D. Baumann, and U. Truyen. 2006. Diversity within the current algal species Prototheca zopfii: A proposal for two Prototheca zopfii genotypes and description of a novel species, Prototheca blaschkeae sp. nov. Int. J. Syst. Evol. Microbiol. 56:1419-1425.

Rozen, S., and H. J. Skaletsky. 2000. Primer3 on the WWW for general users and for biologist programmers. Pages 365-386 in Bioinformatics Methods and Protocols: Methods in Molecular Biology. S. Krawetz and S. Misener, ed. Whitehead Institute for Biomedical Research/Humana Press, Totowa, NJ.

Satoh, K., K. Ooe, H. Nagayama, and K. Makimura. 2010. Prototheca cutis sp. nov., a newly discovered pathogen of protothecosis isolated from inflamed human skin. Int. J. Syst. Evol. Microbiol. 60:1236-1240

Xu, H. B., R. H. Jiang, W. Sha, L. Li, and H. P. Xiao. 2010. PCR-single-strand conformational polymorphism method for rapid detection of rifampin-resistant Mycobacterium tuberculosis: Systematic review and meta-analysis. J. Clin. Microbiol. 48:3635-3640. 